\title{
Civilisations
}

Revue internationale d'anthropologie et de sciences

humaines

42-1 | 1993

Amérique latine-Europe

\section{Les ONG et les nouveaux défis de la coopération au développement de la Communauté européenne}

\section{German Granda}

\section{OpenEdition \\ Journals}

Édition électronique

URL : http://journals.openedition.org/civilisations/2109

DOI : 10.4000/civilisations. 2109

ISSN : 2032-0442

\section{Éditeur}

Institut de sociologie de l'Université Libre de Bruxelles

\section{Édition imprimée}

Date de publication : 1 novembre 1993

Pagination : 191-202

ISBN : 0009-8140

ISSN : 0009-8140

Référence électronique

German Granda, «Les ONG et les nouveaux défis de la coopération au développement de la Communauté européenne », Civilisations [En ligne], 42-1 | 1993, mis en ligne le 30 novembre 1996 consulté le 19 avril 2019. URL : http://journals.openedition.org/civilisations/2109; DOI : 10.4000/ civilisations. 2109

Ce document a été généré automatiquement le 19 avril 2019.

(c) Tous droits réservés 


\title{
Les ONG et les nouveaux défis de la coopération au développement de la Communauté européenne
}

\author{
German Granda
}

1 Malgré les événements survenus après la signature du traité de Maastricht, mais dans le cadre d'une réflexion approfondie sur le contenu du traité, la volonté politique des pays est de progresser dans les différents thèmes qui y ont été approuvés.

\section{Une Europe plus solidaire avec les pays du Sud}

2 L'Union européenne cherche essentiellement à promouvoir un progrès économique et social équilibré et durable, par la création d'un espace sans frontières intérieures, et à affirmer l'identité européenne sur la scène internationale par le développement d'une politique extérieure et de sécurité commune.

3 Il est incontestable que l'Europe communautaire ne souhaite pas se replier sur elle-même, d'autant plus que sa présence sur la scène internationale est fondamental pour contribuer à la paix, la démocratie, le progrès et la solidarité dans le monde, valeurs essentielles de cette identité européenne. C'est pourquoi elle vise également à renforcer l'unité et la cohésion de sa politique extérieure et, par conséquent, sa politique de coopération au développement, pièce maîtresse de la politique extérieure.

4 Selon le traité de Maastricht, la politique de coopération communautaire est complémentaire de celle des Etats membres et doit contribuer spécialement au développement économique et social durable des pays en voie de développement (PVD), à la consolidation de la démocratie et de l'Etat de droit, ainsi qu'au respect des droits de l'homme et des libertés fondamentales.

Dans cette tâche, la coopération non gouvernementale (ONG) joue un rôle essentiel en unissant les divers acteurs de la société civile européenne dans les efforts de coopération avec les PVD, en tenant compte du fait que nous vivons dans un monde de plus en plus 
interdépendant où, face à des problèmes tels que la dégradation de l'environnement, l'immigration, la lutte contre la drogue, les déséquilibres dans le commerce international, etc., il faut des solutions communes et partagées entre le Nord et le Sud.

\section{Portée de la coopération communautaire}

6 Les changements survenus dans les pays d'Europe de l'Est et le conflit au Moyen-Orient ont amené la Communauté à faire face à de nouvelles responsabilités internationales et ont suscité de grandes expectatives. Dès le début, la Communauté s'est mobilisée pour contribuer au renforcement de la sécurité et de la stabilité en Europe.

Mais l'Europe communautaire n'en néglige pas pour autant (et ne négligera pas) ses engagements de coopération avec les pays du Sud. Elle a donc continué à renforcer ses programmes de coopération avec l'Afrique, l'Asie et l'Amérique latine, accordant une importance particulière à la coopération non gouvernementale.

8 La coopération avec les pays ACP progresse grâce à l'accord de Lomé IV, malgré les graves difficultés politiques, économiques et sociales auxquelles sont confrontés la majorité des pays. Dans un climat de dialogue et d'engagement, la quasi totalité des pays a déjà fixé ses programmes indicatifs, en donnant la priorité au développement rural, aux infrastructures socio-économiques, à la protection de l'environnement, au rôle de la femme, etc.

9 Les pays du Sud Méditerranée, liés à l'Europe par de puissants liens historiques et géographiques, connaissent aujourd'hui des problèmes importants: conflits politicoreligieux, forte croissance démographique, taux élevé de chômage des jeunes surtout, etc. Ces problèmes ont une répercussion en Europe, si l'on tient compte de leur grande influence, tant sur la sécurité de la région que sur la société européenne, en raison de la pression migratoire croissante et de l'indispensable intégration sociale. Consciente de ces problèmes, la Communauté a renforcé sa coopération financière et technique avec les pays cités.

10 La coopération avec l'Amérique latine a aussi été renforcée au cours des dernières années, favorisée par le rétablissement de la démocratie dans presque tous les pays. Récemment, la Communauté a approuvé un nouveau règlement pour la coopération avec les pays d'Amérique latine et d'Asie, dont les orientations mettent l'accent sur la dimension humaine et structurelle du développement: problèmes démographiques, appui aux secteurs de l'économie informelle, programmes sociaux qui bénéficient aux groupes les plus touchés par les politiques d'ajustement structurel, etc. Les ressources financières ont été augmentées en même temps que l'on étendait la durée des actions par la réalisation de programmes pluriannuels.

11 Si la situation de nombreux pays du Sud reste caractérisée par l'aggravation de la pauvreté pour une grande majorité de la population, qui ne participe pas aux structures de décision et de pouvoir ni aux bénéfices du développement, chaque pays est fondamentalement responsable de l'élaboration et de l'exécution de sa propre stratégie de développement. Toutefois, il incombe à la $\mathrm{CE}$ de contribuer aux efforts que doivent accomplir les pays du Sud en matière de réforme et de démocratisation de l'Etat pour une meilleure participation de la société civile. 


\section{Droits de l'homme et démocratie}

12 La résolution adoptée par le Conseil de l'Europe (novembre 91) met l'accent sur la priorité et la cohésion que la Communauté et ses Etats membres doivent apporter aux actions positives de promotion des Droits de l'homme et de la démocratie. Le Parlement européen participe aussi activement à ces nouvelles orientations ; il a en effet approuvé, à partir de cette année, une nouvelle ligne budgétaire pour la promotion des Droits de l'homme et de la démocratie dans les PVD, qui vient s'ajouter à celle qui existe déjà pour l'Amérique latine.

13 Les Droits de l'homme constituent un aspect important de la coopération communautaire, dont il faudra tenir compte dans la conception et la mise en oeuvre des nouveaux projets et programmes de développement. Dans ce sens, deux axes importants d'actions positives sont: d'une part, le renforcement de l'Etat de droit et de ses institutions démocratiques et, d'autre part, le renforcement de la société civile et du tissu démocratique, en s'appuyant sur les processus de décentralisation administrative et la participation des différents secteurs de population dans les programmes de développement.

Cette nouvelle ligne budgétaire ouvre également un large champ de collaboration entre la CE et les ONG. Leurs organisations peuvent, précisément par leur expérience sur le terrain et leur proximité avec les différents secteurs de population des PVD, contribuer à la réalisation de projets qui permettent d'atteindre ces objectifs. Dans ce sens, les ONG ont à jouer un rôle important de soutien aux mouvements associatifs liés aux initiatives locales, que ce soit dans des programmes de développement gouvernemental ou non gouvernemental, à caractère rural ou urbain. La CE a donc un intérêt particulier à soutenir des projets d'ONG de développement qui comprennent un aspect de démocratisation, permettant de la renforcer au niveau de la base et de la promotion des Droits de l'homme.

Il est important également de souligner les nouvelles possibilités qu'offre la coopération décentralisée, aspect novateur de Lomé IV qui, par l'appui à divers secteurs (pouvoirs publics décentralisés, associations locales, organisations professionnelles, ONG, etc.), cherche à renforcer la participation de la société civile aux processus démocratiques des pays ACP. A cet égard, le Parlement européen a, sur proposition de la Commission, approuvé récemment une nouvelle ligne budgétaire élargissant la coopération décentralisée au reste des PVD.

\section{La coopération de la CEE avec les ONG}

La collaboration avec les ONG pendant 17 ans présente des résultats assez positifs et s'est intensifiée de manière importante au cours des dernières années. La preuve en est sa participation accrue dans de nouveaux domaines de la coopération communautaire, ainsi que l'augmentation annuelle des ressources financières que la Communauté canalise à travers ces organisations. Ainsi, en 1991, les ressources ont augmenté de façon significative pour atteindre 480 millions d'ECU (MECU), c'est-à-dire $50 \%$ de plus qu'en 1990. 
Cette contribution communautaire aux diverses actions des ONG est répartie comme suit : (en MECU)

- 93,8 pour le cofinancement de petites actions de développement dans PVD (art. budgétaire B7-5010) : augmentation de $16 \%$ par rapport à 1990 ;

- 10,3 pour le cofinancement d'actions de sensibilisation de l'opinion publique européenne sur les questions de développement (art. B7 -5010) : représente une augmentation de $11 \%$ par rapport à 1990 ;

- 185,1 pour l'aide alimentaire (chapitre 72 du budget) : augmentation de $51 \%$ par rapport à 1990 ;

- 4,5 pour l'achat de produits alimentaires par les ONG (art. B7-5011) une diminution de $14 \%$ par rapport à 1990 ;

- 79,7 pour l'aide d'urgence (art. B7-5000) : augmentation de $21 \%$ par rapport à 1990 ;

- 10,9 destinés aux réfugiés et personnes déplacées (art. B7-302) : augmentation de $6 \%$;

- 5,0 pour aider les ONG intervenant au Chili en appui au processus démocratique ainsi que pour contribuer à la coopération économique avec ce pays (art. B7 -5073) : même montant qu'en 1990 ;

- 5,4 pour la lutte contre la drogue (art. B7-5045) avec $46 \%$ d'augmentation par rapport à 1990 ;

- 60,0 pour l'aide aux victimes de l'apartheid (art. B7-5070) soit le double du budget de 1990;

- 10,7 pour les populations des Etats de la « ligne du front » (Namibie, Botswana,Mozambique, etc.) (art. B7-5071) : une diminution de $19 \%$;

- 10,0 pour aider les ONG intervenant en Cisjordanie et Gaza (art. B7-406) : une augmentation des deux tiers par rapport à 1990 ;

- 2,0 pour aider les ONG intervenant au Vietnam (art. B7-5074) : nouvelle ligne budgétaire ;

- 0,5 pour aider les ONG intervenant au Cambodge (art. B7-5075) : nouvelle ligne budgétaire.

Un domaine important de collaboration entre la CE et diverses ONG européennes spécialisées est constitué par l'aide d'urgence. En 1991, plus de $40 \%$ des fonds communautaires destinés à cette aide ont été mobilisés par les ONG. La Communauté doit faire face à un nombre croissant de situations d'urgence partout dans le monde : l'appui pour lutter contre l'épidémie de choléra au Pérou ; l'aide aux victimes de la violence dans des pays comme le Soudan, la Somalie, le Liberia, l'Éthiopie, etc.; l'aide suite aux catastrophes naturelles comme les cyclones et les inondations au Bangladesh : telles ont été quelques-unes des destinations de l'aide communautaire en 1991. Ce type d'actions requiert rapidité et efficacité, conformément aux nécessités.

Cela a incité la Commission à créer un Bureau européen d'aide humanitaire d'urgence, qui sera doté de moyens permanents susceptibles d'être mobilisés dans les plus brefs délais. Pour ce type d'aides, la Commission continuera à appliquer dans toutes ses actions le principe de base de non-discrimination de populations et de pays.

Une coordination plus étroite avec les Etats membres, ainsi qu'une meilleure complémentarité avec les Nations Unies constitueront deux bases essentielles de cette action. De même, on établira une plus grande coordination avec les ONG, pour laquelle ces organisations devront faire les efforts nécessaires pour faciliter cette tâche. 


\section{Appuyer les initiatives des partenaires du Sud et sensibiliser les citoyens européens}

21 Tout en respectant l'autonomie, le pluralisme et la spécificité des ONGD, la CE cofinance des projets de développement dans les PVD ainsi que des actions de sensibilisation de l'opinion publique en Europe. Les ONGD européennes qui introduisent une demande de cofinancement auprès de la $\mathrm{CE}$, doivent satisfaire un certain nombre de critères pour être éligibles. L'ONGD en question doit être autonome, sans but lucratif, elle doit avoir son siège dans un Etat membre et la majorité de ses ressources humaines et financières doivent être d'origine européenne. Les modalités de cofinancement sont diverses. En général, la contribution de la $\mathrm{CE}$ est fixée à $50 \%$ du coût total d'une action et ne peut dépasser 150.000 ECU par an pour les actions dans les PVD (avec un maximum de 500.000 ECU en 5 ans) et de 80.000 ECU pour les actions de sensibilisation ( 3 ans et 240.000 ECU maximum).

Globalement, les différents types d'actions cofinancées au titre de ce programme ont bénéficié entre 1976 et 1991 de 643 MECU dont $91 \%$ sont allés à des cofinancements d'actions dans les PVD et $9 \%$ à des actions de sensibilisation en Europe.

La CE contribue au cofinancement des projets, en général de petite taille, en faveur et avec la participation active des secteurs de populations pauvres dans des domaines qui contribuent à satisfaire leurs besoins fondamentaux (alimentation, éducation, santé, etc.). Les actions d'appui institutionnel aux ONG du Sud et organisations de base constituent aussi une priorité du programme communautaire. De 1976 jusqu'à 1991, la contribution cumulée de la CE se chiffre à 586 MECU. Cette somme a permis la réalisation de 4.474 projets, gérés par 509 ONG dans 120 PVD. Ils ont été réalisés dans les pays de l'Afrique subsaharienne (42\%), de l'Amérique Latine (33\%), de l'Asie (19\%), du Bassin méditerranéen (5\%) et des pays du Pacifique (1\%).

\section{a) Populations et pays bénéficiaires}

La richesse des initiatives des partenaires du Sud dans presque tous les PVD se constate encore une fois à travers la diversité des actions cofinancées en 1991.

Les actions touchent l'ensemble des secteurs de population les plus démunis allant des petits agriculteurs aux travailleurs du secteur informel, en passant par les jeunes marginaux, les communautés paysannes, les associations villageoises, les enfants de la rue, etc.

Les 501 actions de développement cofinancées en 1991 ont été réalisées dans 104 pays en développement.

La ventilation géographique des contributions communautaires à ces actions se présente comme suit :

\begin{tabular}{|l|l|l|l|l|}
\hline & MECU & 1991 & 1990 & 1989 \\
\hline & & $\%$ & $\%$ & $\%$ \\
\hline
\end{tabular}




\begin{tabular}{|l|l|l|l|l|}
\hline Afrique subsaharienne & 36,8 & 39,3 & 40,5 & 43,5 \\
\hline Amérique latine & 37,5 & 40,0 & 32,0 & 33,0 \\
\hline Asie & 12,9 & 13,8 & 21,5 & 18,0 \\
\hline Méditerranée & 5,7 & 6,0 & 6,0 & 4,0 \\
\hline Pacifique & 0,9 & 0,9 & - & 1,5 \\
\hline & 93,8 & 100 & 100 & 100 \\
\hline
\end{tabular}

La répartition entre pays $\mathrm{ACP}$ et pays non-ACP était la suivante :

\begin{tabular}{|l|l|l|l|l|}
\hline & MECU & 1991 & 1990 & 1989 \\
\hline & & $\%$ & $\%$ & $\%$ \\
\hline Pays ACP & 41,5 & 44 & 41 & 44,5 \\
\hline Pays non-ACP & 52,3 & 56 & 59 & 55,5 \\
\hline & 93,8 & 100 & 100 & 100 \\
\hline
\end{tabular}

On peut tirer quelques commentaires à partir des deux tableaux ci-dessus ainsi que sur base de la liste des projets cofinancés dans les dernières années et en 1991.

D'une part, la tendance à la baisse des demandes concernant l'Afrique subsaharienne s'est ralentie par rapport à 1990, tandis qu'elle a été forte pour l'Asie. D'autre part, on observe une importante augmentation des demandes en faveur de l'Amérique latine en 1991. Il est difficile de donner des raisons exhaustives pour expliquer ces évolutions puisque les variations dans la répartition géographique d'une année à l'autre sont le plus souvent conséquence d'un grand nombre de facteurs. Néanmoins on peut signaler certains éléments explicatifs de l'ensemble des évolutions. Ainsi, dans le cas de l'Afrique, malgré les graves problèmes économiques et sociaux d'une grande partie des populations, divers conflits politiques, des dictatures et guerres civiles ont limité dans certains pays les possibilités des organisations de base pour accomplir des projets de développement. $\mathrm{Au}$ contraire en Amérique latine, en plus d'une importante tradition des mouvements et organisations sociales, les processus de démocratisation dans la majorité des pays ont favorisé le travail des ONG et des organisations de base.

Si l'on observe révolution par pays durant les dernières années, quelques pays d'Afrique tels que le Zimbabwe, le Burkina Faso, le Bénin, le Burundi, le Cameroun ont bénéficié d'une augmentation des contributions communautaires, tandis que l'appui à des projets en Mauritanie, Somalie, Soudan a diminué. 
Répartition par état membre des fonds alloués aux ONGD en 1991

\begin{tabular}{|c|c|c|c|c|c|c|}
\hline \multirow[b]{2}{*}{ Etats membres } & \multicolumn{2}{|c|}{$\begin{array}{l}\text { Actions de développement } \\
\text { dans les PVD. }\end{array}$} & \multicolumn{2}{|c|}{$\begin{array}{l}\text { Actions de sensibilisation } \\
\text { de lopinion publique } \\
\text { europénne }\end{array}$} & \multicolumn{2}{|l|}{ Total } \\
\hline & $\begin{array}{l}\text { Nombre } \\
\text { d'uctions }\end{array}$ & $\begin{array}{l}\text { Contribution CE: } \\
\text { en ECU }\end{array}$ & \begin{tabular}{l|} 
Nombre \\
d'actions
\end{tabular} & $\begin{array}{l}\text { Contribution CE } \\
\text { en ECU }\end{array}$ & $\begin{array}{l}\text { Nombre } \\
\text { dactions }\end{array}$ & $\begin{array}{l}\text { Contribution CE } \\
\text { en ECU }\end{array}$ \\
\hline Belgique & 68 & 10989040 & 18 & 853148 & 86 & 11842188 \\
\hline Danemark & 4 & $\$ 13894$ & 5 & 438361 & 9 & 952255 \\
\hline Espagne & 39 & 7408789 & 9 & 413651 & 48 & 7822420 \\
\hline Allemagne & 84 & 18408113 & 18 & 1497841 & 102 & 19905954 \\
\hline France & 75 & 12818174 & 17 & 937434 & 92 & 13755608 \\
\hline Grèce & 3 & 654048 & 1 & 221340 & 4 & 875388 \\
\hline Irlande & 10 & 1523227 & 4 & 300025 & 14 & 1823252 \\
\hline Italie & 80 & 15425594 & 16 & 1756314 & 96 & 17181908 \\
\hline Luxemboury & 21 & 927212 & & & 21 & 927212 \\
\hline Pays-Bas & 14 & 2182205 & 14 & 1351129 & 28 & 3533334 \\
\hline Portugal & 4 & 482646 & 2 & 108657 & 6 & 591303 \\
\hline Royaume-Uni & 90 & 15952946 & 19 & 1870302 & 110 & 17823248 \\
\hline Consortium ONG & & & & & & \\
\hline $\begin{array}{l}\text { de plusieurs Etats } \\
\text { membres }\end{array}$ & 9 & 6497736 & 2 & 534200 & 11 & 7031936 \\
\hline Total & 501 & 93783604 & 125 & 10282402 & 627 & $104066006^{*}$ \\
\hline
\end{tabular}

* il convient d'ajouter à ce chiffre des divers pour $325827 \mathrm{ECU}$ tendance à la hausse. D'une part, certains pays tels que l'Argentine, l'Uruguay et, dans une moindre mesure, le Venezuela n'étaient pas traditionnellement demandeurs de cofinancement auprès des ONGD. Cependant, la crise économique de ces dernières années a forcé la population à s'organiser pour répondre aux besoins croissants des plus défavorisés. Il en est de même pour les pays andins (Colombie, Bolivie, Pérou, Équateur), dans lesquels les difficultés économiques s'aggravent suite à l'application des politiques d'ajustements structurels. Cette situation de crise a pour conséquence d'engendrer un accroissement permanent du nombre de marginalisés et bien entendu des besoins. l'Amérique centrale ont diminué de manière généralisée ces trois dernières années. traditionnellement, les principaux bénéficiaires traditionnels, ont diminué ces deux dernières années.

\section{b) Éducation au développement} différents groupes cibles européens (grand public, décideurs politiques, médias, organisations professionnelles, jeunes, etc.) sur différents thèmes de développement (commerce, dette, environnement, drogue etc.) avec une approche d'interdépendance Nord-Sud. Par les contacts directs avec leur partenaires des PVD et par leur rôle dans la société civile, les ONGD sont bien placées pour mobiliser et sensibiliser les Européens sur leur responsabilité dans le développement des pays du Sud. Entre 1979 et 1991 la CE a cofinancé 898 actions de sensibilisation pour un montant global de 56,9 MECU dans les douze Etats membres. 


\section{Environnement et développement} communautaire, pour devenir l'un des objectifs prioritaires de l'Union européenne, dans le contexte du développement durable. Cela implique que les exigences en matière d'environnement devront être intégrées dans la définition et la mise en oeuvre d'autres politiques de la Communauté. aujourd'hui ont de plus en plus une dimension planétaire; ils exigent donc des solutions au même niveau. Bon nombre de ces problèmes, dans les pays du Nord, mais plus encore dans ceux du Sud, menacent gravement l'avenir de leurs processus de développement. Il est donc nécessaire d'intégrer, une optique de développement et d'interdépendance Nord-Sud tant dans les causes que dans les solutions possibles à ces problèmes. PVD, tels que la désertification (dans les pays d'Afrique) et la situation des forêts tropicales (particulièrement la forêt amazonienne), dans une optique de développement intégral. La Communauté contribue par différents canaux financiers à la réalisation d'actions dans ces domaines. Cette année, en effet, les montants des lignes budgétaires pour l'écologie et les forêts tropicales ont été augmentés de manière significative.

40 Lomé IV vise à atteindre un développement durable et soutenable en intégrant systématiquement l'environnement aux autres secteurs prioritaires. De nombreux pays ACP ont incorporé cet objectif dans leurs programmes-cadres. On prévoit également des actions spécifiques pour l'environnement dans toute une série de domaines (désertification, gestion des ressources naturelles, protection des zones côtières, reboisement, etc.) et, d'autre part, on inclut l'interdiction d'exporter des déchets dangereux ou radioactifs.

41 La Conférence des Nations Unies sur l'Environnement et le Développement (CNUED), qui s'est tenue en juin 1992 à Rio de Janeiro, a permis à la Communauté de réaffirmer son engagement en faveur d'un style de développement durable et de contribuer à résoudre les problèmes auxquels sont actuellement confrontés les PVD. La Commission et ses Etats membres ont exprimé leur volonté politique par la récente résolution approuvée par le Conseil (décembre 91), dans laquelle on a tenté d'adopter une position coordonnée et cohérente pour la Conférence de Rio.

Il convient de souligner l'intérêt et les préoccupations des ONG dans ce domaine et les efforts importants qu'elles ont déployés à la conférence de l'ONU pour élaborer un plan d'action commun pour les ONG. Elles réalisent aussi un travail très positif sur le terrain. Ainsi, dans le cadre du développement rural, elles mènent des actions avec les communautés indigènes pour récupérer leur identité et leurs traditions agricoles, en favorisant un développement harmonieux qui contribue à la préservation de l'environnement. Avec les secteurs urbains, elles soutiennent des programmes d'assainissement, de recyclage des déchets et de lutte contre la pollution dans les grandes villes où se concentre de plus en plus une population pauvre d'origine rurale.

Dans ce sens, les ONG réalisent des actions importantes en tant qu'exécuteurs de projets de coopération communautaire au niveau bilatéral, permettant d'atteindre un effet 
multiplicateur important au profit des populations des PVD, et d'utiliser plus efficacement les ressources communautaires investies.

\section{Réflexions finales} projets et s'ouvrir davantage au cumul de nouvelles initiatives qui proviennent du Sud, à la recherche d'un développement plus participatif. durable et équitable.

\section{RÉSUMÉS}

The article deals with the specifie question of the Community cooperation for the development in Latin America. The autors evaluates the new forms of cooperation that have progressively developed for the past years. Beyond conventions, it is the concept of cooperation for development in itself that suffers a deep transformation. Political cooperation or decentralised cooperation enable to envisage a new approach where objectives such as consolidation of democracy or environment protection have a priority place in comparison to strictly economic prospects. In particular, the implantation of cooperation through new operators, European and Latino-American NGO's, entails coherence between the actors of the different private companies and their implication in the definition and the realisation of development projects doser to concrete demands of local populations 